\title{
La retroalimentación formativa una práctica eficaz en tiempos de pandemia
}

\author{
Formative feedback an effective practice in times of pandemic
}

O feedback formativo é uma prática eficaz em tempos de Pandemia

Mariluz Francisca Huayhua Prada

conternurita@gmail.com

https://orcid.org/0000-0002-5999-2441

Universidad Cesar Vallejo, Lima-Perú

\author{
Carmen del Pilar Avila Zamudio \\ pilarcamucha@gmail.com \\ https://orcid.org/0000-0002-8769-3019 \\ Universidad Cesar Vallejo, Lima-Perú
}

\author{
Yolanda Clara Vargas Pacherres \\ yola_vp@hotmail.com \\ https://orcid.org/0000-0001-5337-2690 \\ Universidad Cesar Vallejo, Lima-Perú
}

Carmen Rosa Buitron Bruno

carmenrosabuitron@gmail.com

https://orcid.org/0000-0001-7673-5378

Universidad Cesar Vallejo, Lima-Perú

Artículo recibido 30 de agosto 2021, arbitrado y aceptado 22 de septiembre 2021 y publicado 15 de diciembre 2021

\section{RESUMEN}

El periodo lectivo 2020-2021, de acuerdo al Ministerio de Educación son años a típicos en la escuela peruana, se tuvo que implementar el sistema de educación remoto o a distancia, por esta razón las escuelas afrontaron la situación retadora de llevar a este contexto la manera de cómo se impartió el servicio educativo, continuar con el desarrollo curricular de contenidos priorizados y realizar la evaluación formativa, en esta línea de acuerdo al nuevo contexto que asumió la educación peruana el objetivo de este estudio fue explorar desde la literatura académica como se aborda el tema de la retroalimentación formativa dentro de la práctica pedagógica, se empleó como método el enfoque cualitativo de revisión bibliografía, tomando como referencia artículos científicos de revistas indexadas, se concluyó que, la retroalimentación formativa es una alternativa potente para valorar los desempeños de los estudiantes.

Palabras clave: Aprendizaje; Docente; Escuela; Evaluación; Retroalimentación

ABSTRACT

RESUMO

The 2020-2021 school period, according to the Ministry of Education are typical years in the Peruvian school, the remote or distance education system had to be implemented, for this reason the schools faced the challenging situation of bringing the way of how the educational service was delivered, continue with the curricular development of prioritized contents and carry out the formative evaluation, in this line according to the new context that Peruvian education assumed, the objective of this study was to explore from the academic literature how the On the subject of formative feedback within pedagogical practice, the qualitative approach of bibliographic review was used as a method, taking as reference scientific articles from indexed journals, it was concluded that formative feedback is a powerful alternative to assess the performance of students.

Key words: Learning; Teacher; School; Evaluation; Feedback
O período letivo 2020-2021, de acordo com o Ministério da Educação são anos típicos na escola peruana, o sistema de educação a distância ou a distância teve que ser implantado, por isso as escolas enfrentaram o desafio de trazer o caminho de como o atendimento educacional foi entregue, continuar com o desenvolvimento curricular dos conteúdos priorizados e realizar a avaliação formativa, nesta linha de acordo com o novo contexto que a educação peruana assumiu, o objetivo deste estudo foi explorar a partir da literatura acadêmica como a Sobre a temática formativa. feedback dentro da prática pedagógica, utilizou-se como método a abordagem qualitativa de revisão bibliográfica, tomando como referência artigos científicos de periódicos indexados, concluiu-se que o feedback formativo é uma alternativa poderosa para avaliar o desempenho dos alunos.

Palavras-chave: Aprendizagem; Professor; Escola; Avaliação; Feedbac 


\section{INTRODUCCIÓN}

La aparición del COVID-19 cambió el sistema educativo a nivel mundial, para proteger la salud de los ciudadanos, se determinó el confinamiento obligatorio o cuarentena, después se pasaría al proceso de emergencia sanitaria, en tal sentido los países tuvieron que implementar medidas para adaptar el sistema educativo a esta nueva realidad. En el Perú el 6 de abril de 2020 se normó como inicio del año escolar y se estableció la educación remota o a distancia, tanto en instituciones de gestión pública o privada, podía ser sincrónica o asincrónica, desde el Ministerio de Educación (MINEDU) se implementó la plataforma de libre acceso "Aprendo en casa" la cual se complementó con la emisión de los contendidos mediante programas televisivos y radiales.

Con respecto a los contenidos curriculares en el año 2020 se priorizaron los temas relacionados a salud y el manejo de las emociones, para lo cual el Minedu promulgó la RV $\mathrm{N}^{\circ} 093$ (MINEDU, 2020) "Orientaciones pedagógicas para el servicio educativo de Educación Básica durante el año 2020 en el marco de la emergencia sanitaria por el Coronavirus COVID-19"; este documento tuvo como recomendaciones, las labores a implementar desde la gestión institucional, desempeño docente, apoyo de los padres, etc. además para fortalecer la evaluación de los aprendizaje se indicadores de logros necesarios en cada nivel de estudio, también se promulgó la RV N 94 (MINEDU, 2020) "Norma que regula la Evaluación de las Competencias de los Estudiantes de Educación Básica”.

Entre las múltiples exigencias que significó implementar el nuevo sistema educativo, surgieron las dificultades de adaptación por parte de los distintos actores de la comunidad educativa, el principal fue la falta de competencias digitales en los docentes, les costó emplear adecuadamente al principio las Tecnologías de la Información y la Comunicación (TIC), e igual situación sucedió con los estudiantes los cuales a pesar de ser nativos digitales, dominaban el empleo de redes y carecían de habilidades para las plataformas y sistemas de conectividad que se empezaron a utilizar, por otro lado los padres tuvieron que cumplir la labor de acompañar a sus hijos en el desarrollo de sus experiencias de aprendizaje y actividades de extensión, ante estos acontecimientos la función más importante la afrontaron los directivos desde su gestión, tuvieron que asumir estrategias para adecuar a la comunidad educativa y afrontar el tema de las emociones que generó la pandemia (Gonzales-Sánchez et al., 2021).

Ante lo expuesto la forma cómo se implementó el sistema educativo necesitó de pautas que ya se empleaban durante la forma presencial, debido a que se continuo con la evaluación formativa, fue necesario una adecuada retroalimentación para evidenciar en los estudiantes que estaban llegando a los indicadores de logros esperados, o brindarles recomendaciones necesarias en sus aprendizajes, es por eso que se describirá cuáles son las concepciones que se tiene de este tema con respecto a retroalimentación.

De acuerdo a Anijovich (2019) el docente se comunica con los estudiantes y las familias, recoge las evidencias de aprendizaje los analiza, lo interpreta y busca encontrar los aspectos logrados $\mathrm{y}$ los que hay que fortalecer, luego elabora un comentario en el que describe lo que el estudiante aprendió y lo que necesita mejorar para brindar 
un acompañamiento, un apoyo adecuado; de esta manera la retroalimentación nos da a conocer las fortalezas, oportunidades de mejora, (Stobart, 2010) ayuda al estudiante a comprender su manera de aprender y permite que el docente reflexione sobre su propuesta de enseñanza.

Desde otra perspectiva Quezada y Salinas (2021) propusieron un modelo de retroalimentación donde el estudiante recogeinformación sobre su desempeño fomentando su capacidad de autorregulación que le permite ser un aprendiz autónomo y el docente ser un mediador, siguiendo la misma línea Muñoz (2020) realizó un análisis sobre los tipos o modos de retroalimentación que ejecutan los docentes en el área de matemática para ello aplico el modelo teórico Mathematics Teacher Speialisedd Knowledg (MTSK) señalando que los docentes poseen una sólida formación pedagógica para que puedan realizar una óptima retroalimentación respecto a las metas de aprendizaje.

Tomando los aportes de Manresa y Ramos (2021) quienes investigaron sobre el impacto de la retroalimentación que brinda el docente a los estudiantes sea virtual o presencial podrán lograr la autorregulación, autoevaluación, coevaluación y reflexión sobre el aprendizaje. En esta línea, para lograr un cambio en el estudiante se debe considerar durante la retroalimentación que el docente de a conocer el objetivo de la tarea, considerar el progreso que realiza el estudiante al ejecutar la actividad aplicando una estrategia, posteriormente el nivel de autorregulación se evidencia a través del compromiso del estudiante (Cabrera, 2020; Manresa y Ramos, 2021).

Según Llerena et al., (2020) ejecutar la retroalimentación por parte de los docentes consolida los aprendizajes que no quedaron claros en los estudiantes de educación, tomaron como ejemplo a los alumnos de educación inicial, esta labor la realizan de manera conjunta con los padres ya que ellos son los que refuerzan el aprendizaje creando espacios de reflexión. Sin embargo, Asiú Corrales et al., (2021) sostuvieron que la retroalimentación es fundamental para consolidar los aprendizajes y Llanos y Tapia (2021) contribuyen en la autoconfianza y San Andrés Soledispa et al., (2021) señalaron que es una estrategia para mejorar los aprendizajes.

La retroalimentación fortalece la práctica profesional, y el desempeño académico de los estudiantes, tomando los aportes de la zona de Vygotsky de la zona próxima, citado por (Gonzales et al., 2014) es la distancia que existe entre la zona de desarrollo real y zona de desarrollo potencial, en este espacio se da la interacción entre docente y estudiante a través de la retroalimentación brindando soporte, ayuda detectando los errores, señalando aciertos para brindar orientación y estimular al estudiante en su aprendizaje (Canabal y Margalef, 2017) también se reorienta la práctica pedagógica del docente ajustando sus estrategias, recursos didácticos reformula sus metodologías, para el logro de aprendizajes permitiendo que los estudiantes realicen las tareas con diversos niveles de dificultad hasta adquirir su autonomía (Chávez, 2001; Onrubia, 1999).

Como afirmó Ramaprasad 1983 citado por (Wiliam, 2009; García, 2015) al retroalimentar se brinda información que ayude al estudiante a reducir la distancia de su desempeño actual y el objetivo o aprendizaje deseado. Esa información explica cómo está yendo ante los esfuerzos que el estudiante realiza 
para lograr los objetivos y como puedes hacer para acercarte más a él. Mientras que para (Sadler, 1989) existen tres condiciones para que los estudiantes se beneficien de la retroalimentación es:1) que conozcan y comprendan la meta de aprendizaje que se pretende alcanzar, 2) comparen su nivel actual de desempeño con el ideal o deseado, 3) establecer acciones que le permitan alcanzar el nivel de desempeño esperado, la retroalimentación permite a los docentes diagnosticar a los estudiantes, saber dónde se encuentran y hacían donde se debe ir y que se puede hacer para llegar a esa meta, para ello el docente puede hacer uso de rubricas de calificación o presentar ejemplos, brindar oportunidad a los estudiantes de realizar correcciones necesarias, reforzar el contenido que no quedo claro.

Durante el proceso de retroalimentación, resultó necesario compartir los propósitos de aprendizaje que orienta al estudiante hacia dónde van, por qué y para qué, (Hattie y Timperley, 2007) también cuales son los criterios de evaluación de esta manera permitirá que el estudiante se autoevalúe (Muñoz, 2020; Lima, 2017) esto le permite saber dónde está con relación a los logros de aprendizaje y hacia donde tiene que llegar aumentando su responsabilidad ante su propio aprendizaje, ante ello el docente reorienta su práctica pedagógica y retroalimenta a los estudiantes en función a sus necesidades. Por ello, es necesario compartir con los estudiantes los propósitos aprendizaje y los criterios de evaluación porque permite saber que tan lejos o cerca esta con respecto a los competencias o desempeños a lograr.

El aporte de Stobart (2010) quien manifestó que, la retroalimentación direcciona al estudiante lo que tiene que hacer de esta manera cierra las brechas entre lo que realizan, el modo que se desempeña durante el aprendizaje y las expectativas de logro. Por otro lado, (Lozano y Tamez, 2014) retroalimentar implica tomar acciones en la zona de desarrollo próximo, así la retroalimentación puede entenderse como andamiaje, proporcionando información para disminuir la brecha existente entre los niveles de desempeño actual y el deseado, en este contexto de educación a distancia por la pandemia es necesario coordinar con las familias para que autorice la comunicación con los estudiantes en la ejecución de la retroalimentación ya sea oral o escrita y en el momento adecuado que le permita comprender sus errores para superarlos y le ayude a mejorar su desempeño.

Habiendo definido líneas atrás algunos aportes desde la literatura académica sobre la retroalimentación, en necesario comprender el rol del docente al ejecutar esta labor, para Anijovich (2019) son interacciones dialogadas entre estudiantes y docentes, Llanos y Tapia (2021) cuya finalidad es articular las evidencias de aprendizaje con los objetivos y los criterios de evaluación. Al retroalimentar se desarrolla una conversación, en un clima de confianza y respeto, Quiñones et al., (2021) formulando preguntas y repreguntas con la finalidad de ayudar al estudiante a que comprenda como es su modo de aprender, a valorar sus procesos y su resultado, autorregulando su aprendizaje. Por ello se replantea una manera de relacionarse y comunicarse con los estudiantes. Desde el punto de vista del estudiante al retroalimentar no solo aprende contenidos sino también comprende que y como mejorar, explicando las estrategias que le ayudan a aprender, usando adecuadamente una variedad de instrumentos que le favorecen a mejorar el aprendizaje, esto le conlleva a asumir 
responsabilidades y convertirse en autónomo (Anijovich y González, 2011)

Es importante la función que desempeña el docente en la retroalimentación, también acompaña y guía al estudiante hasta que supere sus dificultades, Vásquez (2020) la manera o forma como interactúa con él, expresando sus opiniones, juicios sobre cómo se está desarrollando el aprendizaje con aciertos, errores, fortalezas y debilidades, permitirá que el estudiante se involucre y reflexione, vea los errores como parte de un aprendizaje y ponga el esfuerzo por mejorar haciéndole consciente de lo que deben lograr, de lo que están logrando y de lo que les falta por alcanzar, por ello Falcón et al., (2021) manifiesta que los docentes tienen que tener un contenido disciplinar en sus bases teóricas para poder analizar la información recogida, emitir juicios de valor o valorar las evidencias y registrar la información de manera objetiva. Además, la información que brinda el docente debe ser descriptiva, específica y concreta de cómo está desarrollando en relación a los propósitos de aprendizaje, para que pueda mejorar y corregir sus errores, brindar información primeramente sobre los aspectos positivos para que el estudiante tenga mayor predisposición para recibir las sugerencias y no adopte una actitud defensiva (Asiú Corrales et al., 2021).

Es necesario que el estudiante reciba la retroalimentación en el momento para que pueda incorporar los cambios necesarios redirigiendo su desempeño, Stobart (2010) puntualizó cuando el estudiante desarrolla una tarea difícil es necesario brindar una retroalimentación inmediata con la finalidad de reducir los sentimientos de frustración y si es una tarea sencilla demorar la retroalimentación esto asegurará que reflexione. (Quiñones et al., 2021) sostiene que para lograr mejores aprendizajes los docentes necesitan retroalimentar, cuando el docente devuelve la información sobre el análisis de las producciones tiene que generar reflexión en el estudiante pero muchas veces no sucede así, porque algunos docentes no comunican de manera clara y comprensible el mensaje o porque el estudiante no pone el esfuerzo por comprender la retroalimentación ejecutada, Por consiguiente la eficacia de la retroalimentación se evidencia en la manera como es decepcionada de parte del estudiante y también en la eficacia como es comunicada por el docente. Por estas razones se evidencia estudiantes que aún no logran su autonomía

Se retroalimenta a los docentes como a los estudiantes durante todo el proceso de enseñanza aprendizaje, reformulando y reorientando las acciones del docente y del estudiante permitiendo un desarrollo integral. (Asiú Corrales et al., 2021) En este contexto de pandemia, Tenutto, (2020) es necesario resaltar que la retroalimentación fue una gran oportunidad de aprendizaje: los docentes reflexionan respecto a la interacción que se desarrolla en las prácticas pedagógicas relacionadas con la enseñanza y los estudiantes reflexionan respecto a sus aprendizajes. Asiú Corrales et al., (2021) un elemento que refuerza los aprendizajes y mejora la interacción docente estudiante en tiempos del Covid-19.

Se ha definido que es la retroalimentación, luego se brindó algunos aportes de cómo la desempeñan los docentes, a continuación, se desarrollaran algunas estrategias que se encontró sobre el tema, para Hattie y Timperley (2007) identifica 4 tipos de retroalimentación: 1) A nivel de la tarea, se basa en distinguir que tanto comprendió de la tarea 2) A nivel del proceso de la tarea, se focaliza en las estrategias que realiza el estudiante al proceso 
de su desempeño 3) A nivel de autorregulación, los propios estudiantes de manera independiente dirigen sus acciones desarrollando su autonomía, autocontrol, autodirección y autodisciplina 4) A nivel de la propia persona, destaca las evaluaciones personales y escribe expresiones de motivación hacia los estudiantes.

Tomado los aportes de una de las principales teóricas sobre este tema Anijovich (2019) propuso 5 estrategias para desarrollar la retroalimentación: 1) Ofrecer preguntas, esto permite desarrollar habilidades metacognitivas sobre cómo está aprendiendo. 2) Describir el trabajo del estudiante, es como devolver en espejo lo que el estudiante realizó. 3) Valorar los avances y logros, es necesario para que los estudiantes reconozcan en qué medida y como lo está logrando. 4) Ofrecer sugerencias, aporta reducir la brecha entre él está en que se encuentra y hacia la meta. 5) Ofrecer andamiaje, orienta ayuda acompaña el docente al estudiante hasta que logre los propósitos de aprendizaje. Por otro lado, Wilson, citado por Muñoz (2020) presenta la escalera de la retroalimentación como una de las herramientas que debe ser usada al realizar una retroalimentación precisando 4 estrategias: 1) clarificar, el docente formula preguntas para aclarar una idea permite entender mejor algunos puntos. 2) valorar, se rescata las fortalezas y aspectos positivos. 3) expresar inquietudes evitando hacer criticas agresivas sino preguntas dirigidas a buscar nuevas soluciones 4) sugerir, el docente brinda recomendaciones específicas y concretas para mejorar el trabajo y alcanzar el nivel de desarrollo potencial.

De acuerdo a todo lo expuesto se realizó una revisión teórica de la retroalimentación formativa, en tal sentido, el objetivo de este estudio es explorar desde la literatura académica como se aborda el tema de la retroalimentación formativa dentro de la práctica pedagógica en el contexto de la pandemia del COVID-19. Este estudio se consideró de impacto porque brinda los aportes de la literatura de manera sistemática y organizada, de algunas definiciones más representativas sobre la retroalimentación formativa, asimismo, demostró que desde los aspectos teóricos esta labor se puede desempeñar en el contexto de la emergencia sanitaria originada por el COVID-19 en el cual la educación es remota o a distancia.

\section{MÉTODO}

Para este estudio se empleó el enfoque cualitativo de revisión bibliográfica, para lo cual se recurrió a gestores de información de artículos científicos como Scielo, Scopus y WOS, se priorizó para construir el periodo de la pandemia del COVID-19, las publicaciones del año 2020 y 2021; asimismo, para la búsqueda se tomó en cuenta las palabras clave relacionadas a esta investigación siendo la principal retroalimentación, para contextualizar la información también fue necesario recurrir a documentos normativos sobre educación emitidos por el gobierno peruano.

\section{DESARROLLO Y DISCUSIÓN}

Los artículos revisado brindaron como información relevante sobre la retroalimentación, para Mollo-Flores y Medina-Zuta (2020) que analizaron los desafíos actuales de la evaluación formativa dentro del contexto de incertidumbre por el COVID 19 considerando que: todo docente debe explicar el paradigma del enfoque 
socio constructivista por Vygotsky, que los estudiantes cuenten con un guía capacitado para el proceso de aprendizaje, considera desarrollar la retroalimentación de manera sincrónica y asincrónica, entre el docente y el estudiante, para lograr el autoconocimiento y la autorregulación. Asimismo, Asiú Corrales et al., (2021) sostuvieron que, la evaluación formativa que contribuye a la formación integral del estudiante a través de la retroalimentación, cuyo rol del docente es comunicar los propósitos de aprendizaje, así como la tarea del estudiante es cerrar ese espacio entre lo que sabe y la meta a donde llegar. También, Cabrera (2020) determino la importancia de la retroalimentación, brindando información por medio de sugerencias y comentarios empleando estrategias que promueve la reflexión y autonomía del estudiante en el logro de su aprendizaje. Por otro lado, Cáceres y Tapia (2021) realizaron una revisión de literatura sobre la percepción de los docentes y estudiantes ante la retroalimentación, promoviendo un andamiaje cognitivo para que el estudiante logre los propósitos dependiendo como interactúe y aborde, esto permitirá su desarrollo integral.

Algunas de las investigaciones consultadas coincidieron con el enfoque cualitativo. buscando comprender la realidad que se investigó relacionado con el proceso de la retroalimentación formativa, los trabajos contemplaron diferentes formas de participación de los estudiantes y docentes para saber su percepción sobre la retroalimentación. Desde otra perspectiva, (Quiñones et al., 2021) analizaron que muchos docentes de escuelas rurales no tienen claro el proceso de retroalimentación porque demanda mucho trabajo y tiempo, además, implicaría un acompañamiento constante hacia el trabajo de los estudiantes por lo que resultaría cansado ejecutarlo. Sin embargo, Falcón et al., (2021) plantea que es necesario que los docentes tengan un contenido disciplinar en sus bases teóricas para incorporar la evaluación formativa sobre todo la retroalimentación, esto le permitirá emitir juicios de valor o valorar las evidencias y registrar la información de manera objetiva, se realizó una entrevista a 8 docentes de una institución pública usando una ficha de registro que contenía un cuestionario para las entrevistas, posteriormente se describió las experiencias de aprendizaje generando una reflexión con los mismos docentes llegando a la conclusión que los docentes no están preparados para realizar una retroalimentación efectiva no tienen claro los propósitos de aprendizaje ni los criterios de evaluación.

En otralínea Berlaga y Juárez (2020) investigaron la importancia de emplear un instrumento que mida la manera como se imparte la retroalimentación. lo que le falta aún por comprender. Mientras que, para Muñoz, (2020) fue más importante tomar la opinión de los docentes para comprendes cómo se llevaba este proceso, llegaron a la conclusión que es fundamental que se realice la retroalimentación formativa porque reorienta las acciones dela práctica pedagógica hacia un andamiaje cognitivo para que el estudiante logre los objetivos de aprendizaje potenciando la participación, la autoeficacia y la actitud positiva permitiendo un desarrollo integral.

Más innovador en el contexto de la emergencia sanitaria Manresa y Ramos (2021) analizaron el impacto de la retroalimentación en un entorno virtual que realiza los docentes sobre los estudiantes de Educación Secundaria; sin embargo fue con personas adultas; pero permito inferir que este 
proceso sea para cualquier tipo de alumno, concluyeron que es necesario para los alumnos adueñarse de los objetivos y criterios de evaluación responsabilizándose de la autorregulación, la autoevaluación, coevaluación y reflexión constante sobre el aprendizaje (López, 2021) cuyo propósito fue conocer como los estudiantes de quinto grado utilizaban la retroalimentación formativa para aprender a solucionar problemas de fracciones para ello emplearon, la aplicación Retromath que ayudó a los docentes en su trabajo de retroalimentar, se aplicó entrevistas a los estudiantes participantes así como la videograbación de las situaciones de retroalimentación para su respectivo análisis, acudiendo a la técnica de la observación, determinando que es importante como los estudiantes acogen y utilizan la retroalimentación considerando el error como una oportunidad para lograr aprendizajes.

En cambio, para Llerena et al., (2020) tomando la percepción de otros actores como fueron los padres, al realizar la retroalimentación conjuntamente con los padres ellos refuerzan el aprendizaje en los estudiantes de nivel inicial. En el aporte de San Andrés Soledispa et al., (2021) desde la perspectiva de los estudiantes de educación superior, examinaron que la retroalimentación busca reforzar las habilidades, capacidades y conocimientos a través de estrategias de motivación y de autorreflexión la retroalimentación permite construir los aprendizajes significativos, desarrolla habilidades y destrezas en los estudiantes.

La revisión de la literatura reafirmó que la retroalimentación formativa es una alternativa potente para valorar los desempeños de los estudiantes, el $100 \%$ de las investigaciones coinciden en señalar que la retroalimentación es la base principal donde los estudiantes reconocen sus fortalezas y debilidades, ven como una oportunidad para que el docente promueva la reflexión en el aprendizaje generando un conocimiento más profundo y significativo. Permite verificar los logros de los estudiantes e impulsar el aprendizaje con información de retorno sirviendo como herramienta para el control de calidad. Esta estrategia es fundamental porque permite que el docente y el estudiante interactúen, efectivizándose el autoconocimiento y la autorregulación como bases fundamentales de la transformación y de mejora continua.

Varios de los autores (Quiñones et al., 2021; Falcón et al., 2021) coincidieron en señalar que, la retroalimentación la cual se brinda a los estudiantes en algunos regiones no es formativa, aún se evidencia la persistencia de continuar con las prácticas tradicionales por ello es necesario implementar capacitaciones a los docentes para empoderarse sobre la evaluación formativa de esta manera podrá atender las necesidades de aprendizaje de sus estudiantes reorientando su práctica pedagógica, contextualizando su práctica profesional dentro de la emergencia sanitaria.

Estudios (Manresa y Ramos, 2021; San Andrés Soledispa et al., 2021; Muñoz, 2020) enfatizaron que la retroalimentación ayudó al estudiante a autorregular su aprendizaje, facilita a que reflexione sobre sus actos, reconozca sus fortalezas, sus dificultades, sus limitaciones, identifique los desempeños que tiene que lograr, corrigiendo errores, esto permite que se autoevalúe, coevalúe y heteroevalue logrando desarrollar sus habilidades de pensamiento crítico. 
El artículo revisado permitió inferir los aspectos positivos de la retroalimentación desde distintos puntos de vista como fue la percepción de los padres, docentes y estudiantes.

\section{CONCLUSIÓN}

De acuerdo a la revisión de la literatura académica, se llegó a la conclusión que la retroalimentación formativa es una alternativa potente para valorar los desempeños de los estudiantes esta consiste es comunicar de manera asertiva el desempeño que desarrolla el estudiante respecto a las metas de aprendizaje, de esta manera le permitirá reducir la brecha entre lo comprendido y lo que le falta aún por comprender.

Durante el proceso de retroalimentación los roles del docente y estudiante son fundamentales orientan la ejecución de actividades pedagógicas hacia un andamiaje cognitivo para que el estudiante logre los propósitos de aprendizaje dependiendo como interactúe y aborde, además permitirá el desarrollo integral del estudiante, mientras que el maestro reflexiona redirigiendo $\mathrm{u}$ orientando las acciones de su práctica pedagógica. Los docentes deben contar con un contenido disciplinar que sustenten sus bases teóricas con dominio de las estrategias de retroalimentación para poder analizar la información recogida, emitir juicios de valor y registrar la información de manera objetiva. Además, es necesario el apoyo de los padres en este proceso.

Finalmente, la bibliografía consultada entre los años 2020 y 2021, en el cual estalló la emergencia sanitaria por el COVID-19, evidenció que esta labor aún se puede desarrollar durante la educación a distancia, esto depende de la creatividad del docente, asimismo fortalecer sus competencias digitales y contar con el apoyo de las familias, en tal sentido que pueda ser posible realizar la retroalimentación formativa.

\section{REFERENCIAS}

Anijovich, R. (2019). Orientaciones para la Formación Docente y el Trabajo en el aula: Retroalimentación Formativa Publicación. Summa, 1, 21-113. https://panorama.oei. org.ar/_dev2/wpcontent/uploads/2019/06/ Retroalimentaci\%C3\%B3n-Formativa.pdf

Anijovich,R.,yGonzález,C.(2011).Elcírculovirtuoso dela retroalimentación. In Evaluar para aprender. Conceptos e instrumentos (pp. 23-37). http:// d20uo2axdbh83k.cloudfront.net/20140531/ e22a6869020858435ab5f59ad26bcbf6.pdf

Asiú Corrales, L., Asiú Corrales, A., y Barboza Díaz, O. (2021). Evaluación formativa en la práctica pedagógica: una revisión bibliográfica. Revista Conrado, 17(78), 134-139. https:// conrado.ucf.edu.cu/index.php/conrado/article/ view/1654/1633

Berlaga, M. de la L., y Juárez, L. (2020). Diseño y validación de un instrumento para evaluar la retroalimentación asertiva en educación normal. IE Revista de Investigación Educativa de La REDIECH, 11, e791. https://doi.org/10.33010/ ie_rie_rediech.v11i0.791

Cabrera, J. (2020). La retroalimentación para mejorar el proceso de aprendizaje en el estudiante. Informática Educación y Pedagogía, 10, 70-75. https://revistas.udenar.edu.co/index. $\mathrm{php} / \mathrm{runin} /$ article/view/6136

Cáceres, S., y Tapia, C. (2021). Modelo de retroalimentación para el aprendizaje: Una propuesta basada en la revisión de literatura. Revista Mexicana de Investigación Educativa, 26(88), 225-251

Canabal, C., y Margalef, L. (2017). La Retroalimentación: La Clave Para una evaluación orientada al aprendizaje. Revista de Curricular y Formación Del Profesorado, 149170.http://hdl.handle.net/10481/47669 
Chávez, A. (2001). Implicaciones educativas de la teoría sociocultural de Vigotsky. Revista Educación, 25(2), 59-65. http://www.redalyc. org/articulo.oa?id=44025206

Falcón, Y., Aguilar-Hernando, J., Luy-Montejo, C. A., y Morillo-flores, J. (2021). La evaluación formativa ¿Realidad o buenas intenciones? Estudio de caso en docentes del nivel primario. Propósitos Representaciones, 9(1). http:// revistas.usil.edu.pe/index.php/pyr/article/ view/1041/1416

García, E. (2015). La evaluación del aprendizaje: de la retroalimentación a la autorregulación. El papel de las tecnologías. RELIEVE. Revista Electrónica de Investigación y Evaluación Educativa, 21, 1-24. http://www.redalyc.org/ articulo.oa?id=91643847005

Gonzales, N., Eguren, M., y De Belaúnde, C. (2014). Desempeño docente y aprendizaje: una aproximación a las prácticas pedagógicas del maestro peruano. Revista de Investigación. https://www.cies.org.pe/sites/default/files/files/ articulos/economiaysociedad/02-eguren.pdf

Gonzales-Sánchez, a., Trujillo, N., Pelayo, I. y Ocaña-Fernández, Y. (2021). Management in strengthening social- emotional competence during health emergencies. Revista Tempos e Espaços Em Educação, 14(33), e15111. https:// doi.org/10.20952/revtee.v14i33.15111

Hattie, J., y Timperley, H. (2007). EL poder de la retroalimentación. Review of Educational Research, 77, 81-112. https://doi. org/10.3102/003465430298487

Lima, G. (2017). Enriquecer la realimentación para consolidar aprendizajes. Virtualidad, Educación y Ciencia, 8(14), 9-26. https://revistas.unc.edu. ar/index.php/vesc/article/view/17328

Llanos M., y Tapia S, (2021). La retroalimentación dialógica: algunos hallazgos en escuelas multigrado. GRADE Grupo de Análisis Para El Desarrollo, 1-47. http://www.grade.org.pe/ creer/archivos/Retroalimentaci\%C3\%B3nLlanos-y-Tapia.pdf
Llerena, A., Sánchez, I., y Hernández, Y. (2020). La retroalimentación y su influencia en la consolidación de aprendizajes en proceso, de niños y niñas de Educación Inicial. Conciencia Digital, 3(3.2), 60-75. https://doi.org/10.33262/ concienciadigital.v3i3.2.1408

López, U. (2021). La retroalimentación en la resolución de problemas fraccionarios, con alumnos de quinto grado de primaria, mediante el uso de una aplicación. Diálogos de Saberes: Investigaciones y Ciencias Sociales, 2007, 1-27. http://dialogossobreeducacion.cucsh.udg.mx/ index.php/DSE/article/view/83 0

Lozano, F., y Tamez Vargas, L. (2014). Retroalimentación formativa para estudiantes de educación a distancia. Revista Iberoamericana de Educación a Distancia., 197-221. http://revistas. uned.es/index.php/ried/article/view/12684

Lozano M., y Tamez, L. (2014). Retroalimentación formativa para estudiantes de educación a distancia. RIED. Revista Iberoamericana de Educación a Distancia. https://doi.org/10.5944/ ried.17.2.12684

Manresa, M., y Ramos, J. M. (2021). ¿Cómo perciben los estudiantes adultos de educación secundaria en línea el feedback de sus docentes? 1-24. https://doi.org/10.15628/holos.2021.12075

MINEDU. (2020a). $\quad \mathrm{N}^{\circ} 093$ RV Aprobar el documento normativo denominado "Orientaciones pedagógicas para el servicio educativo de Educación Básica durante el año 2020 en el marco de la emergencia sanitaria por el Coronavirus COVID-19". https://www.gob. pe/institucion/minedu/normas-legales/535987093-2020-minedu

MINEDU. (2020b). $\quad \mathrm{N}^{\circ} 094$ RV Aprobar el documento normativo denominado "Norma que regula la Evaluación de las Competencias de los Estudiantes de Educación Básica". https:// www.gob.pe/institucion/minedu/normaslegales/541161-094-2020-minedu 
Mollo-Flores, M., y Medina-Zuta, P. (2020). Evaluación formativa propuesta pedagógica integral en tiempos de pandemia. Maestro y Sociedad. Revista Electrónica Para Maestros y Profesores, 17(4), 635-651. https:// maestroysociedad.uo.edu.cu/index.php/MyS/ article/view/5235

Muñoz, M. (2020). Análisis de las prácticas declaradas de retroalimentación en Matemáticas, en el contexto de la evaluación, por docentes chilenos. Perspectiva Educacional, 59(2), 111135. https://doi.org/10.4151/07189729-vol.59iss.2-art. 1062

Onrubia, J. (1999). Enseñar: crear zonas de desarrollo próximo e intervenir en ellas. El Constructivismo En El Aula, 101-123. http://books.google.com/ books?id=BzOef9UlDb4Cypgis $=1$

Quezada, S., y Salinas, C. (2021) Modelo de retroalimentación para el aprendizaje: Una propuesta basada en la revisión de literatura. RMIE [online]. 2021, vol.26, n.88, pp.225-251. http://www.scielo.org. $\mathrm{mx} /$ cgi-bin/wxis.exe/iah/?IsisScript=iah/iah. xisybase $=$ article $\% 5$ Edlibraryyformat $=$ iso. pftylang=eynextAction $=$ lnkyindexSearch $=$ A U y e x p r S a r c h $=$ Q U E Z A D A + CACERES, + SOLEDAD

Quiñones, L., Zárate-Ruiz, G., Miranda-Aburto, E., y Sosa, P. (2021). Enfoque por competencias (EC) y Evaluación formativa (EF). Caso: Escuela rural. Propósitos y Representaciones, 9(1). https://doi.org/https://revistas.usil.edu.pe/ index.php/pyr/article/view/1036/13 10
Sadler, D. (1989). Formative assessment and the design of instructional systems. Instructional Science, 18(2),119-144.https://doi.org/10.1007/ BF00117714

San Andrés Soledispa, E., Macías, F., y Mieles, G. (2021). La retroalimentación como estrategia para mejorar el proceso de enseñanzaaprendizaje. Revista Electrónica Sinapsis, 61(3), 301-333. https://doi.org/10.31857/ s0869803121030085

Stobart, G. (2010). Tiempos de pruebas: los usos $y$ abusos de la evaluación. In Evaluación Educativa. Magis. Revista Internacional de Investigación en Educación, vol. 4, núm. 7, julio-diciembre, 2011, pp. 211-213 https://www. redalyc.org/pdf/2810/281021741011.pdf

Tenutto, M. A. (2020). Aprendizajes y prácticas educativas en las actuales condiciones de epoca COVID 19 (Investigac, Issue June). CIFFYH. https://www.researchgate.net/ publication/352844596_APRENDIZAJESY-PRACTICAS-EDUCATIVAS-EN-LASACTUALES-CONDICIONES-DE-EPOCACOVID-19

Vásquez Jaico, N. O. (2020). Revisión de evidencias y retroalimentación a estudiantes. Orphanet Journal of Rare Diseases, 21(1), 1-9. https:// www.iegr.edu.pe/wp-content/uploads/2020/08/ R E V I S I O N - D E - E V I D E N C I A S - Y RETROALIMENTACION-1.pdf

Wiliam, D. (2009). Una síntesis integradora de la investigación e implicancias para una nueva teoría de la evaluación formativa. Archivos de Ciencias de La Educación, 3(3), 15-44. http:// www.memoria.fahce.unlp.edu.ar/art_revistas/ pr.4080/pr.4080.pdf 